\title{
Effect of Orifice Shape on Flow Behavior and Impingement Heat Transfer
}

\author{
Azusa Kanamori, Munehiko Hiwada*, Kenyuu Oyakawa and Izuru Senaha \\ Department of Mechanical and System Engineering, Gifu University, 1-1, Yanagido, Gifu 501-1193, Japan
}

\begin{abstract}
Impingement jets are widely used in industries because they provide a high heat transfer coefficient near the stagnation region. However, few methods exist for controlling impingement heat transfer. Recently, a peculiar diffusion process called "axis switching" for three-dimensional free jet has begun to attract attention, and there is a novel possibility of control diffusion and mixture process using this phenomenon. In this paper, we report on the effect of non-circular polygonal orifice shapes on impingement heat transfer. In addition, we demonstrate axis-switching phenomenon by using flow visualization with hydrogen bubbles. Orifice configurations are the regular polygons with 3 to 6 sides. Heat transfer experiments covered the distance between the orifice-to-target plate is 4 to 8 and Reynolds number is $5 \times 10^{4}$ and the heat flux is $600 \mathrm{~W} / \mathrm{m}^{2}$. The flow was visualized in Reynolds number 1,500. For a free jet emerging from a regular polygonal orifice, the location of axis-switching phenomenon shifts toward the orifice exit as the number of sides on the orifice is increased. The iso-Nusselt number profile tends to take the shape of a concentric circle farther upstream. However, with a decrease in the number of sides of the orifice, the iso-Nusselt number profile after axis switching remains downstream.
\end{abstract}

Keywords: Jet, flow visualization, vortex, forced convection, impingement heat transfer.

\section{INTRODUCTION}

Impingement jets [1-3] have been used widely in industries, such as in manufacturing processes for glass and steel requiring cooling, heating, and drying, because impingement jets provide a high heat transfer coefficient near the stagnation region. However, methods for controlling impingement heat transfer are generally poor; therefore, many researchers continue to study this phenomenon. In previous work, the existence of a particular diffusion process called "axis switching" has been reported for the case of a non-circular free jet [4-6]. Axis switching is a phenomenon in which the cross section of an asymmetric jet evolves in a manner such that, after a certain distance from the nozzle, the major and the minor axes are interchanged. The controllability of the diffusion or mixture process associated with axis switching of non-circular free jets has recently attracted the attention of many researchers.

A few studies have investigated the effects of the nozzle shape or orifice configuration on the jet. Mi [7] reported the diffusion process in nine jets from orifices of different shapes by measuring centerline velocities. Hiwada [8] reported the effect of the aspect ratio of an elliptic jet on the heat transfer characteristics of a target plate. In recent studies, Quinn [9] conducted experiments on the near-field mean flow and turbulence characteristics of an equilateral triangular turbulent free jet, and Chaudhari [10] investigated the effect of orifice shape on impingement cooling in synthetic jets from orifices of square, circular, and rectangular shapes.

In this paper, we present experimental studies of the effect of orifice configuration on free-jet behavior visualiz-

*Address correspondence to this author at the Department of Mechanical and System Engineering, Gifu University, 1-1, Yanagido, Gifu 501-1193, Japan; Tel: +81-58-293-2531; Fax: +81-58-293-2491; E-mail: hiwada@gifu-u.ac.jp ing the flow using hydrogen bubbles. We also investigated the heat transfer characteristics of an impinging air jet from a non-circular orifice shapes including triangle, square, pentagon, and hexagon. Moreover, we examined the possibility of controlling impinging heat transfer by changing the orifice configuration.

\section{EXPERIMENTAL SETUP AND METHODS}

\section{Experimental Setup}

Fig. (1) shows the experimental apparatus for the air jet. Air was taken in by a blower, then passed through a rectified section, arrived in a pressure chamber, and then issued from an orifice. The rectangular coordinate system used for

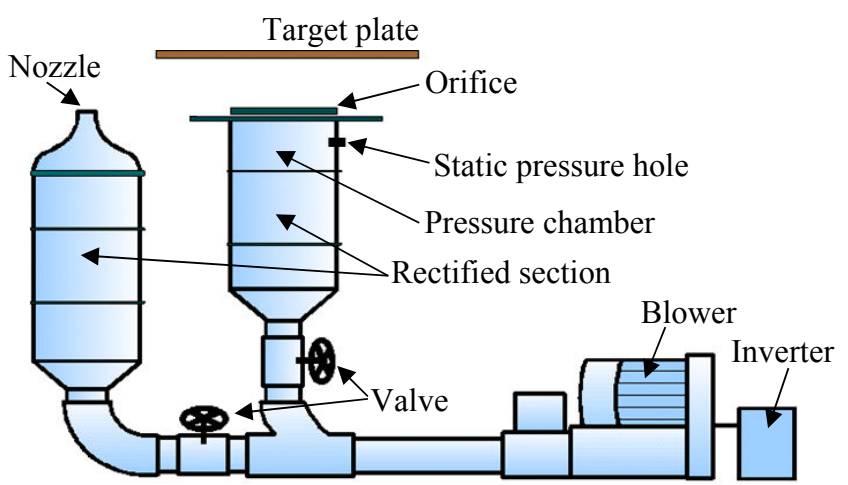

Fig. (1). Experimental apparatus of free jet.

analysis is shown in Fig. (2). Note that the center of the orifice is considered as the origin. The $x$-axis indicates the flow direction along the center of the orifice. The velocity component in the $x$-direction is denoted by $U$, and the 
velocity was measured with an I-type hot wire anemometer. $U_{0}$ is defined as the uniform velocity near the orifice exit. Moreover, the $y_{a^{-}}$and $y_{b}$-axes are defined as the direction from the center to the apical angle and to the base of the orifice, respectively. These directions were defined in order to show the difference in the spread of the jet by the apical angle side and the base side of the orifice. As shown in Fig. (2), $h_{a}$ and $h_{b}$ are defined as the distance from the center to the apical angle and to the base of the orifice, respectively.
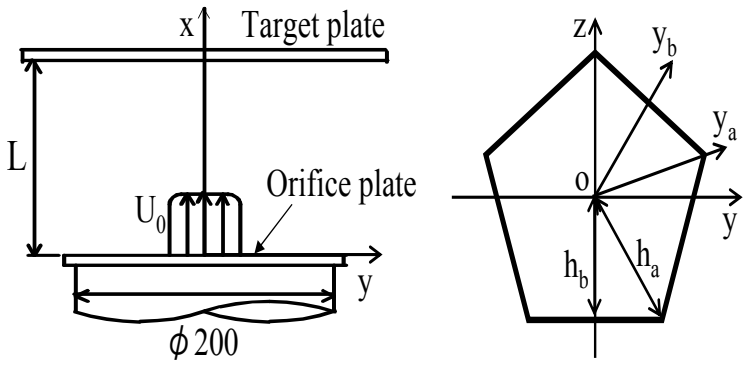

Fig. (2). Experimental apparatus of free jet.

The orifice configurations used were regular polygons as well as a circle. The number of sides on the polygon orifices, $n$, was $3,4,5$, and 6 ; that is, the different configurations of the polygon orifice included a triangle, a square, a pentagon, and a hexagon. Detailed dimensions of each orifice are given in Table 1. The orifice plates were made of 1-mm-thick brass and were fabricated using wire-cutting method in order to have 90 degree relief angles. All polygonal orifices had same area and the equivalent diameter, $D_{e}$, of these was $51 \mathrm{~mm}$. The Reynolds number, Re, was $5.0 \times 10^{4}$ for this experiment using the characteristic length $D_{e}$, and the characteristic velocity $U_{0}$.

\section{Table 1. Orifice Configuration}

\begin{tabular}{|c|c|c|c|}
\hline Configuration & $\mathbf{n}$ & $\mathbf{h}_{\mathbf{a}}[\mathbf{m m}]$ & $\mathbf{h}_{\mathbf{b}}[\mathbf{m m}]$ \\
\hline \hline Triangle & 3 & 39.7 & 19.8 \\
\hline Square & 4 & 32.0 & 22.6 \\
\hline Pentagon & 5 & 29.3 & 23.7 \\
\hline Hexagon & 6 & 28.0 & 24.3 \\
\hline Circle & - & 25.5 & 25.5 \\
\hline
\end{tabular}

The characteristics of the free jet are described by the maximum velocity decay, $U_{m} / U_{0}$, and the turbulence intensity, $T_{u}$, at a specific section, $x / D_{e}$. $U_{m}$ is the maximum velocity along the $x$-direction of the free jet. Note that $T_{u}$ is defined as,

$$
T_{u}=\frac{U_{r m s}}{U_{0}} \times 100
$$

where $U_{r m s}$ is the root-mean-square of the fluctuating velocity in the $x$-direction.

The characteristics of the impingement jet are described by the local wall pressure coefficient on the target plate, $C_{p w}$, and the local Nusselt number on the target plate, $\mathrm{Nu}$, at the orifice-to-target plate spacing, $L / D_{e}$. Note that $C_{p w}$ is defined as,

$$
C_{p w}=\frac{2 p}{\rho \times U_{0}^{2}}
$$

where $\rho$ is the density of dry air and $p$ is the static pressure on the target plate measured with an immersed bell-type precision manometer (ISP-50; Shibata Scientific Technology, Ltd.) having a sensitivity of $0.02 \mathrm{~mm} \mathrm{H}_{2} \mathrm{O}$. The target plate was made of an acrylic resin and measures $700 \mathrm{~mm} \times$ $700 \mathrm{~mm}$. Over 71 pressure taps of diameter $0.8 \mathrm{~mm}$ were placed on its centerline. The center of the orifice was aligned with the center of the target plate.

To measure heat transfer, a stainless-steel foil heater with a surface area of $600 \mathrm{~mm} \times 600 \mathrm{~mm}$ and a thickness of 20 $\mu \mathrm{m}$ was put on a plate made of Bakelite. The foil was divided into five strips to minimize heat loss and maintain a uniform surface heat flux, $q$, of $600 \mathrm{~W} / \mathrm{m}^{2}$ by applying a direct current of $6 \mathrm{~A}$. Heat loss to the back of the target plate can be neglected because the back of the target plate was covered with insulation of glass wool. The surface temperature on the target plate, $T_{w}$, was measured using 38 $\mathrm{Cu}-\mathrm{Co}$ thermocouples that were soldered on the back of the central stainless steel foil strip. The setup of the orifice and the target plate and the measurement method are the same as that for the pressure measurements. The same type of thermocouples measure the free-stream temperature at the orifice exit, $T_{0}$. These thermocouples were connected to an Analog-to-Digital converter (CIO-AD08 and MUX64, with $1 \mu \mathrm{V}$ sensitivity; Omega Engineering, Ltd.) and a personal computer for data acquisition. $\mathrm{Nu}$ is defined as given in the following equation:

$$
N u=\frac{\alpha D_{e}}{\lambda}=\frac{q \times D_{e}}{\lambda\left(T_{w}-T_{0}\right)}
$$

where $\lambda$ is the thermal conductivity of air and $\alpha$ is the local heat transfer coefficient.

\section{Flow Visualization}

Hydrogen bubbles were used for flow visualization. Fig. (3) shows the experimental apparatus used for flow visualization, and Fig. (4) shows the cathode wire setting of the orifice. Hussain [5] reported the deformation of vertical structure on the elliptic jet using flow visualization. Initially, when an elliptic vortex ring rolls up, the plane of the vortex ring is parallel to the nozzle exit plane. Owning to the higher curvature, the major-axis sides move ahead of the minor-axis sides. The vortex configuration becomes like the seams of a tennis ball. Thus, in our flow visualization, two CCD video cameras were used for taking photos from the $x-y$ and $y-z$ planes simultaneously. The frame rate for both cameras was $1 / 30 \mathrm{~s}$. The angle of incidence of light from a lamp was perpendicular to the cameras, that is, the lamp was lit from the $y$-direction. $U_{0}$ for flow visualization was about $0.05 \mathrm{~m} / \mathrm{s}$ and Re was 1,500. A cathode wire of $70 \mu \mathrm{m}$ diameter was made of constantan and was set on the inner edge of the orifice. 


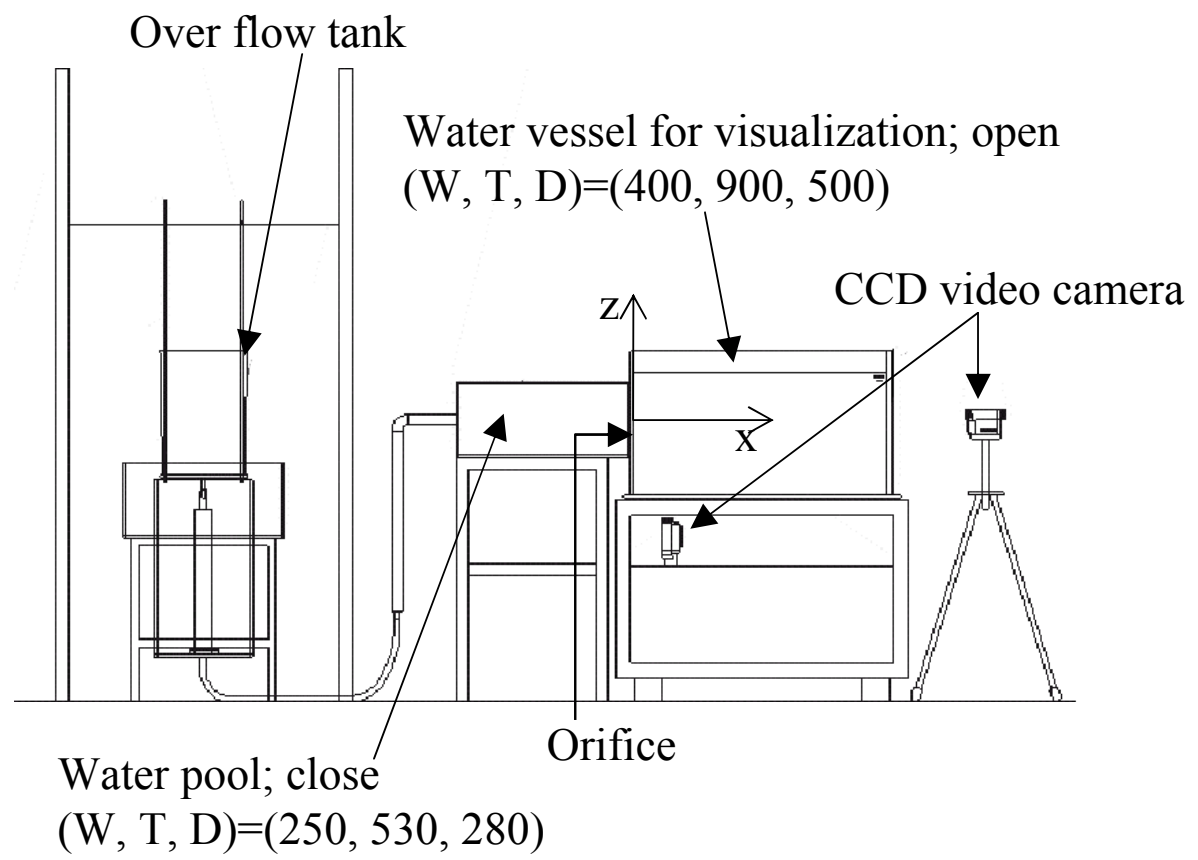

Fig. (3). Experimental apparatus for flow visualization.

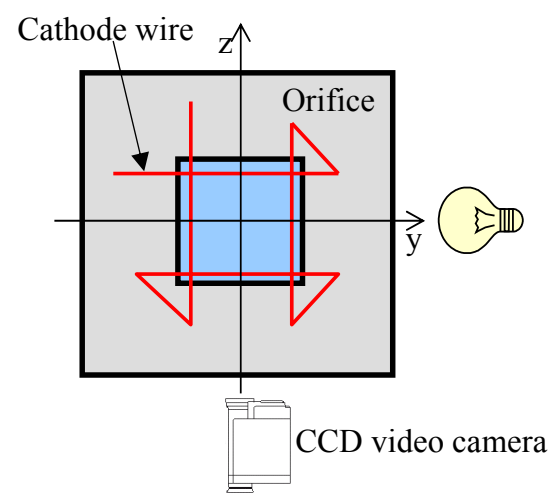

Fig. (4). Cathode wire setting.

\section{EXPERIMENTAL RESULTS AND DISCUSION}

\section{Maximum Velocity Decay and Turbulence Intensity along the Center of the Free Jet}

Fig. (5) shows $U_{m} / U_{0}$ and $T_{u}$ profiles along the center of each regular polygon orifice in the free jet. In addition, the results for a circular nozzle are shown as solid lines. The smaller the number of sides of the orifice, the more upstream the maximum velocity decayed, but the gradient for the velocity decay was nearly the same in all cases. The $U_{m} / U_{0}$ profile for the hexagonal orifice was nearly the same as that of the circular orifice. In the free jet near every orifice exit, $U_{m} / U_{0}$ became larger than one because of the contraction

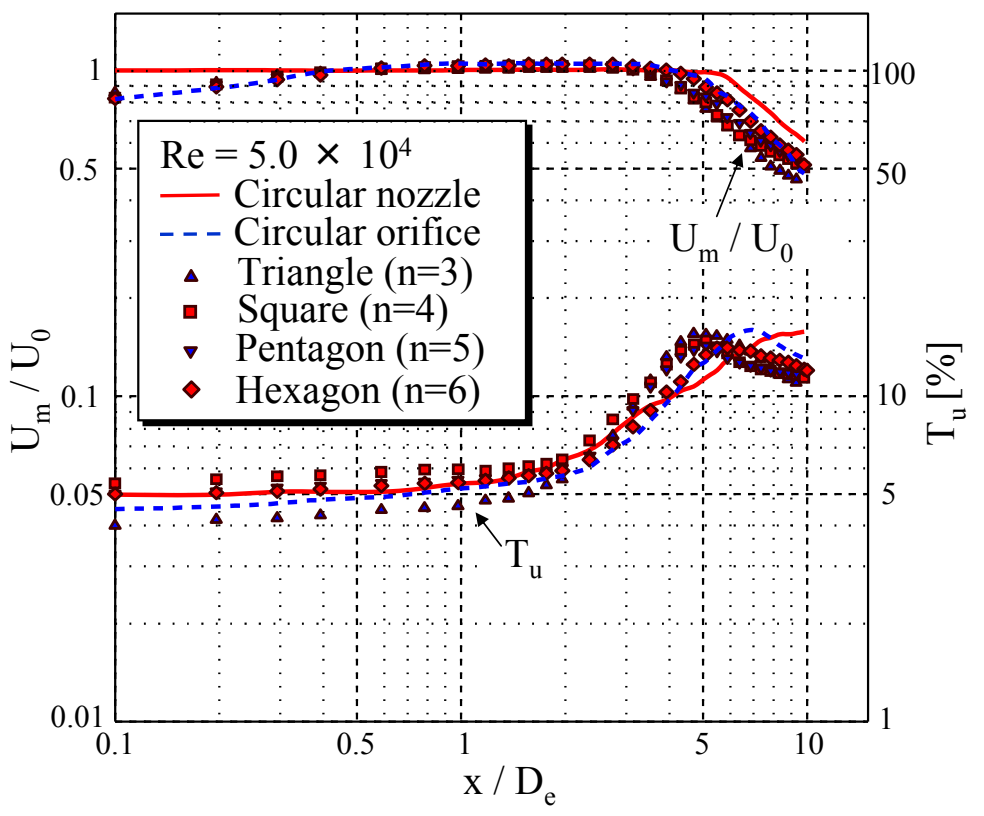

Fig. (5). Maximum velocity delay and turbulence intensity. 


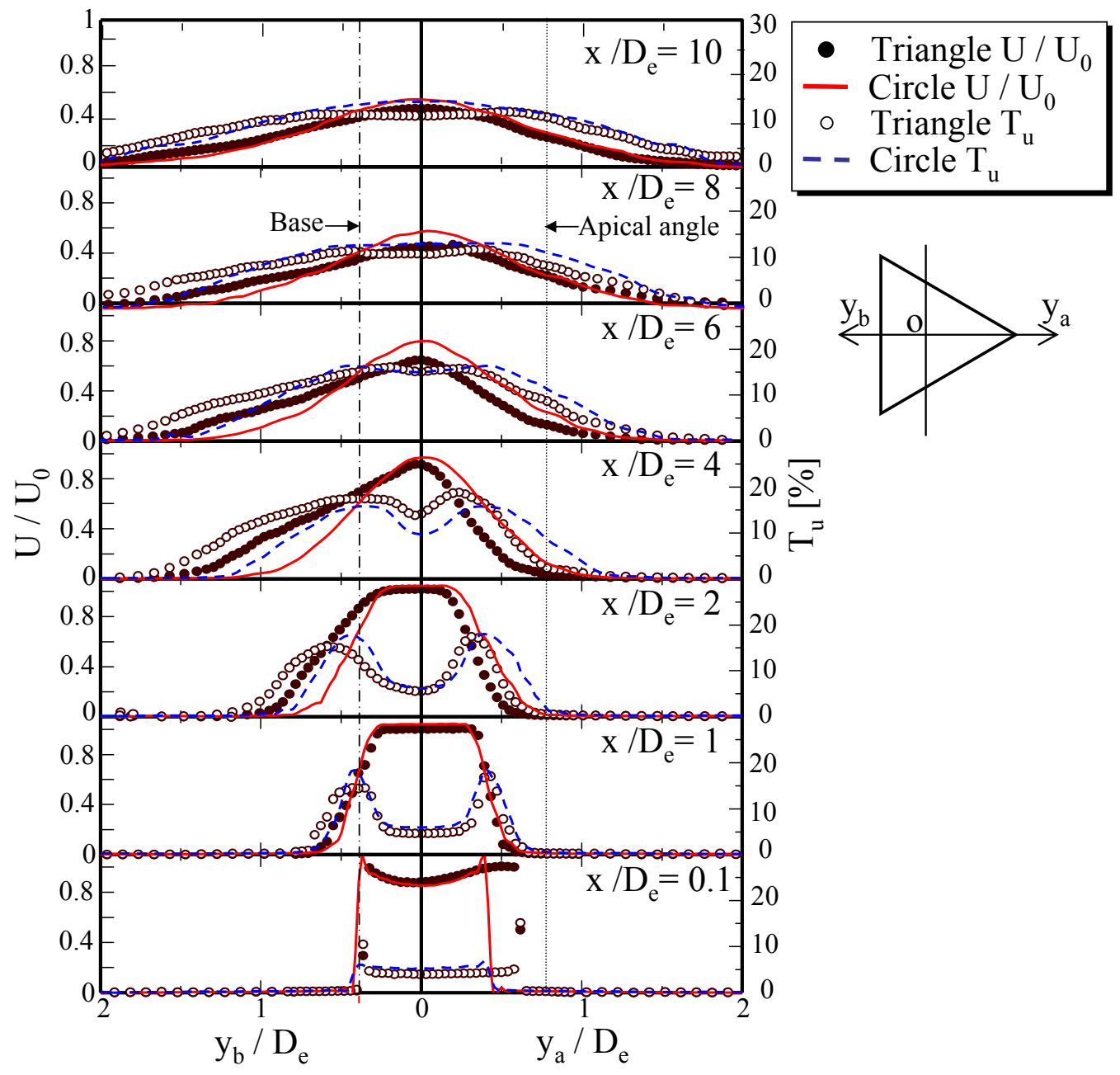

Fig. (6). Velocity and turbulence intensity profiles for regular triangular orifice for $\mathrm{Re}=5.0 \times 10^{4}$.

peculiar to the orifice. The contraction is a phenomenon that is the cross section of the jet become smaller than that of the orifice. With regard to changes in $T_{u}$, the smaller the number of sides of the orifice, the more upstream the $T_{u}$ value increased, and the larger the maximum value of $T_{u}$ observed.

Fig. (6) shows $U / U_{0}$ and $T_{u}$ profiles on specific $x / D_{e}$ for the regular triangular orifice, as a typical example, and for the circular orifice. The profiles of $y_{a} / D_{e}$ and $y_{b} / D_{e}$ are shown on the right side and the left side of the figure, respectively, and a dotted line and a long-dashed shortdashed line indicate the position of $h_{a}$ and $h_{b}$ for the regular triangular orifice, respectively. In the case of the regular triangular orifice at $x / D_{e}=0.1$, the velocity gradient near $h_{b}$ differs from that near $h_{a}$, and the velocity near the apical angle was remarkably low. In addition, the velocity near the orifice edge was faster than that near the center of the free jet, and this was caused by the contraction of the orifice jet. Farther downstream, the $U / U_{0}$ profile spread less between the $y_{a^{-}}$and $y_{b}$-directions, and the $U / U_{0}$ profile of the triangular orifice appeared more like that of the circular orifice. In the case of the circular orifice, the potential core region, where the maximum velocity was maintained in the main stream, was observed in the range $0 \leqq x / D_{e} \leqq 4$; however, in the triangular orifice, the potential core region vanished before $x / D_{e}=4$. With regard to changes $T_{u}$, in the upstream region $0 \leqq x / D_{e} \leqq 4$, for the regular triangular orifice, the maximum value of $U / U_{0}$ in the $y_{b}$-direction was smaller than that in the $y_{a}$-direction. However, the spread of the $T_{u}$ profile in the $y_{b}$-direction was larger than that in the $y_{a^{-}}$ direction.

Based on the $U / U_{0}$ profile on specific $x / D_{e}$, the difference of the spread between the apical angle and the base side for the free jet from the orifice was examined. Halfwidth velocities for the apical angle and the base side of the orifice, $y_{a 1 / 2}$ and $y_{b 1 / 2}$, for the regular triangular and the circular orifices are shown in Fig. (7a). The $U / U_{0}$ profile in Fig. (6) spread more in the $y_{a}$-direction at $x / D_{e}=0.1$, but farther downstream, it becomes larger in the $y_{b}$-direction. The half-width velocities in Fig. (7a) also confirmed these variations. Moreover, over the range $0 \leqq x / D_{e} \leqq 2$ in Fig. (6), the spread width of the $U / U_{0}$ profile in the $y_{a^{-}}$and $y_{b^{-}}$ directions was reversed, thus the $y_{a 1 / 2} / D_{e}$ and $y_{b 1 / 2} / D_{e}$ intersected at $x / D_{e}=0.8$ in Fig. (7a). The intersection point defines the location of axis switching.

Although axis switching was most pronounced in the case of the regular triangular orifice, it was also seen for all the regular polygon orifices like Figs. (7b-d). The intersection point was shifted farther upstream with increasing $n$. In addition, the velocity half-width of all the orifices tended to remain the same as that of the circular orifice in the case of large $x / D_{e}$. 


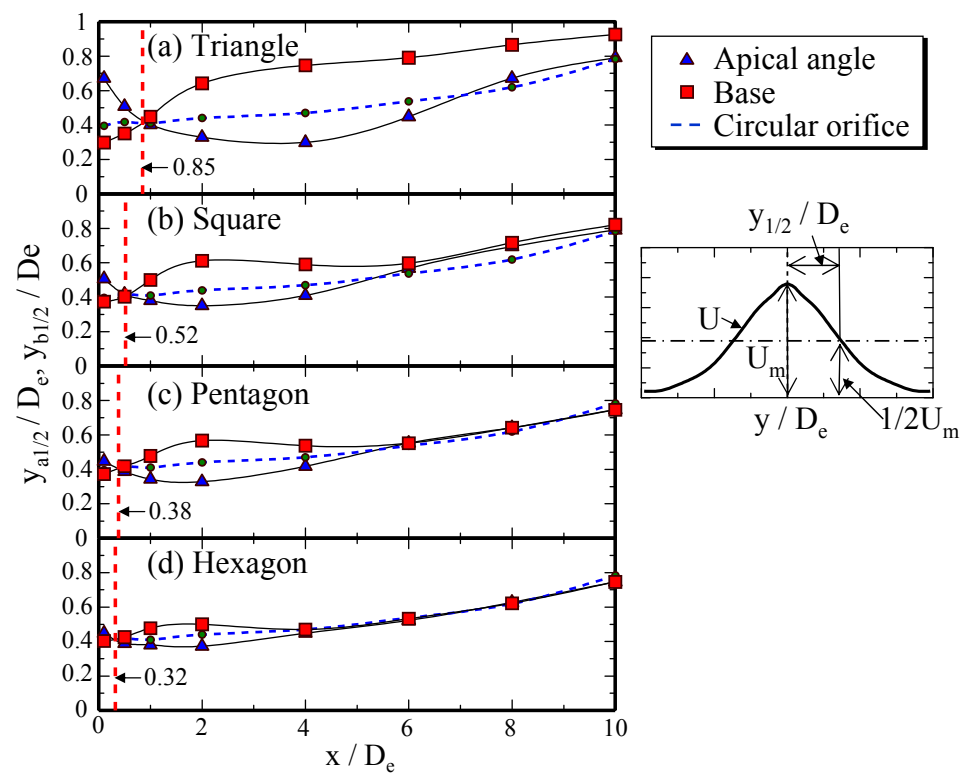

Fig. (7). Velocity half-width profiles for $\mathrm{Re}=5.0 \times 10^{4}$, (a) Triangle, (b) Square, (c) Pentagon, (d) Hexagon.

\section{Flow Visualization of Free Jet using Hydrogen Bubbles}

Photographs of the flow visualization using hydrogen bubbles in the $y-z$ and $x-y$ planes are shown in Figs. (8a-d) for regular triangular, square, and hexagonal, as well as circular orifices. Numbers of the left side in figures were frame numbers of the CCD video camera. Vortex ring

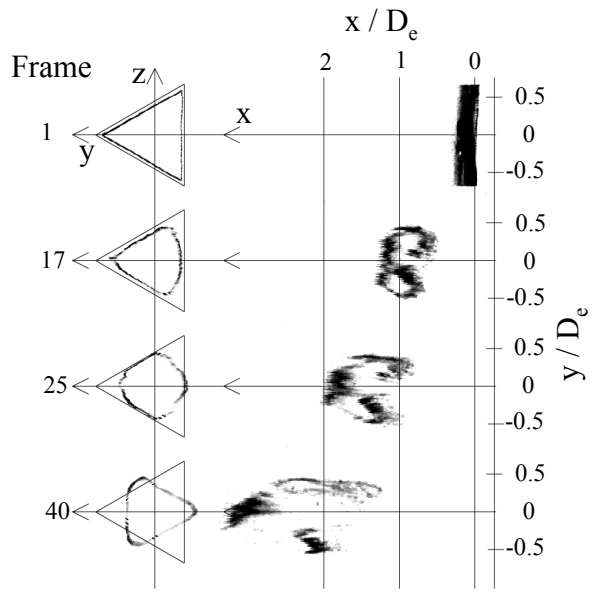

(a) Triangle

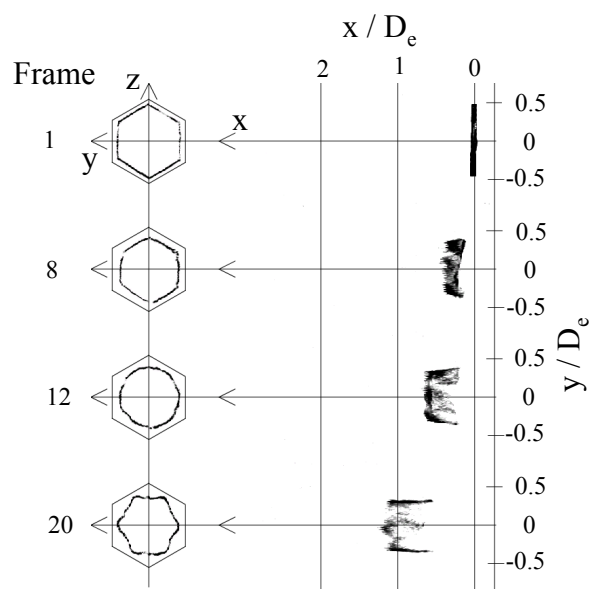

(c) Hexagon

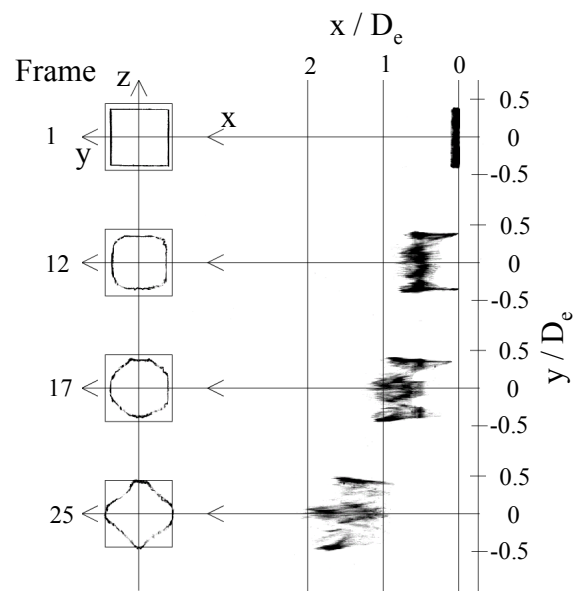

(b) Square

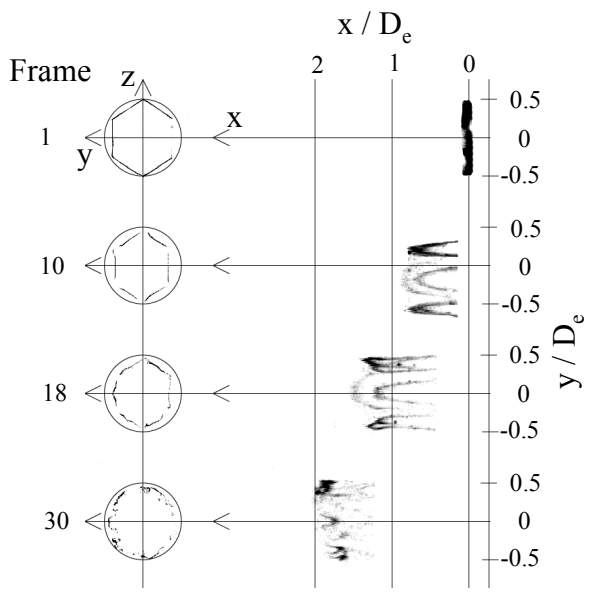

(d) Circle

Fig. (8). Flow visualization results for various orifice shapes for $\mathrm{Re}=5.0 \times 10^{4}$, (a) Triangle, (b) Square, (c) Hexagon, (d) Circle. 


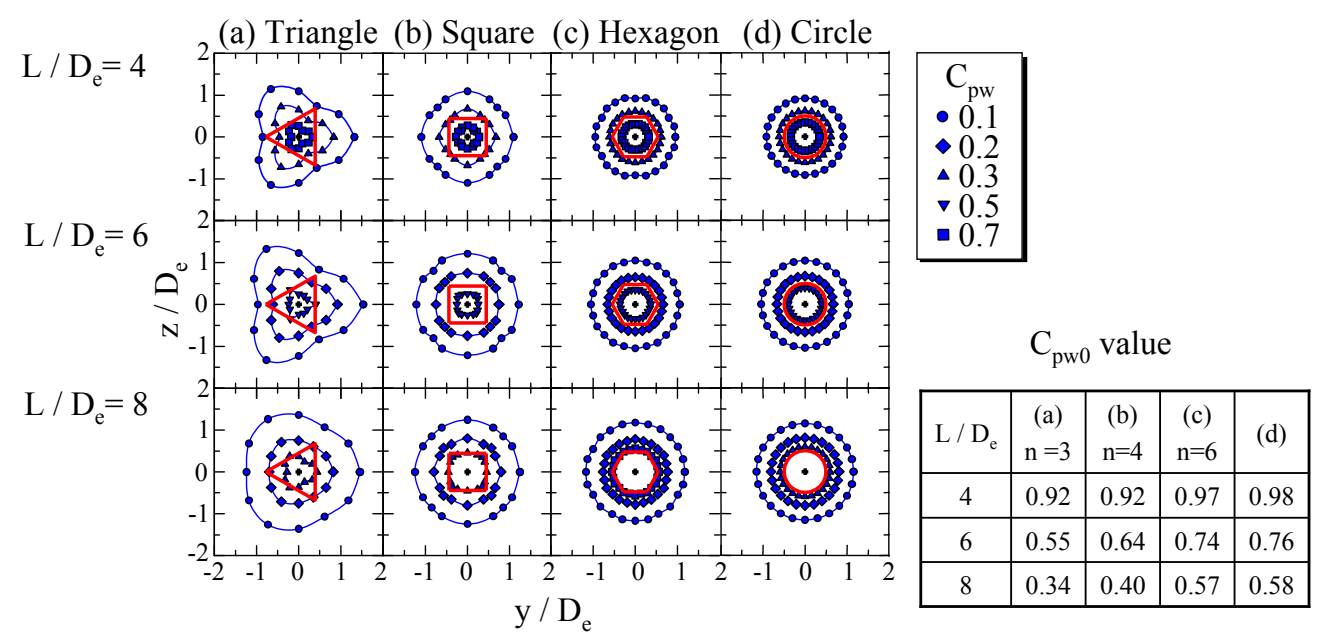

Fig. (9). Iso-local wall pressure coefficient profiles on the target plate, (a) Triangle, (b) Square, (c) Hexagon, (d) Circle.

formed at the orifice exit rolled up with entraining bubble tracers. The images show four typical cross sections: (1) just after the orifice exit, (2) when bubble tracers in the parts of corresponding to the orifice corners have roundness in the $y$ $z$ plane because of the first vortex ring deformation leading to axis switching, (3) when bubble tracers show a nearly circular shape in the $y-z$ plane, and (4) after axis switching, i.e., when the shape of the bubble tracers in the $y-z$ plane reversed after the orifice configuration.

For all orifice configurations, first, vortex ring at the apical angle side with the large curvature began to deform. Then the relative velocity in the $x$-direction at the deformed parts increased more than that of the non-deformed parts. Thus, the part deformed largely on the $y-z$ plane, that is, the apical angle side appears to move forward on the $x-y$ plane more than the base side. This partial difference in the relative velocity of the vortex ring generated other local curvatures of the vortex ring, and the bubble tracers continued deforming accordingly. Moreover, the vortex ring shape changed such that the orifice shape reversed by interchanging the base side and the apical angle side on the $y$ - $z$ plane, and one cycle of axis switching phenomenon was completed. Locations at the occurrence and completion of axis switching shifted down- stream with decreasing $n$, as confirmed by the visualization results (Fig. 7).

\section{Impingement Jet}

To show the planar $C_{p w}$ profile on the target plate, iso- $C_{p w}$ profiles on the $y-z$ plane are shown in Figs. (9a-d). The orifice configurations are the same as that shown in Fig. (7). $C_{p w 0}$ indicates the value of $C_{p w}$ at $y=z=0$, which is the centerline of each orifice on the target plate. For the triangular and square orifices, the shape of the iso- $C_{p w}$ line reversed for $L / D_{e}=4$, thus confirming the occurrence of axis switching. The shape of the iso- $C_{p w}$ line for the triangular orifice was reversed even at $L / D_{e}=8$; however, the iso- $C_{p w}$ line for the square orifice is almost the same as for the circular orifice at the same value of $L / D_{e}$. On the other hand, axis-switching phenomenon was not evident for the hexagonal orifice because the iso- $C_{p w}$ profiles did not show a difference between the hexagonal orifice and circular orifice. These results indicate that axis switching for the triangular orifice produced remarkable effects downstream. Notably, $C_{p w 0}$ increased with increasing $n$ for constant values of $L / D_{e}$, and $C_{p w 0}$ decreased further downstream for the same orifice configuration.

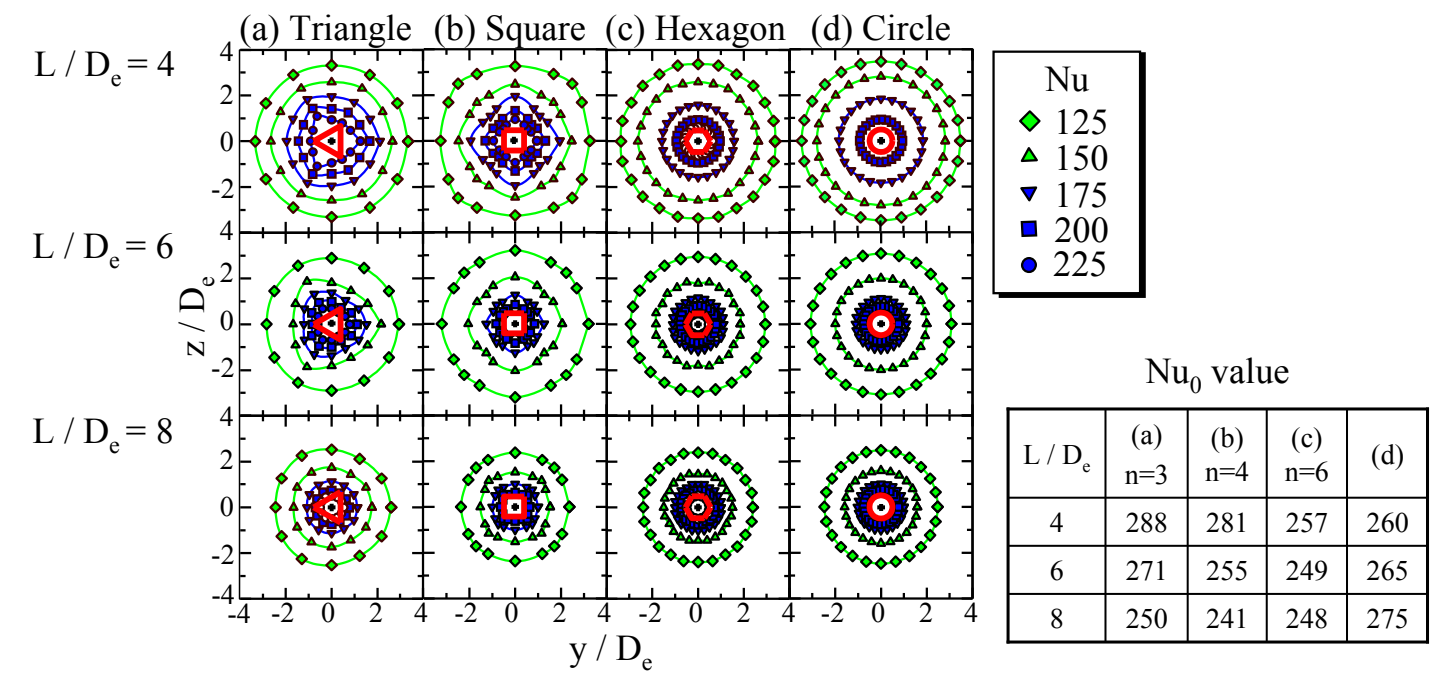

Fig. (10). Iso-local Nusselt number profiles on the target plate, (a) Triangle, (b) Square, (c) Hexagon, (d) Circle. 
To show the planar Nu profile on the target plate, iso- $\mathrm{Nu}$ profiles on the $y-z$ plane are shown in Figs. (10a-d). The orifice configurations and $L / D_{e}$ values are the same as those in Fig. (9). $\mathrm{Nu}_{0}$ indicates the value of $\mathrm{Nu}$ at $y=z=0$, which is the centerline of each orifice on the target plate. The tendencies of iso-Nu profiles in the impingement region, $|y|$ $D_{e}|| z,\left|D_{e}\right|<2$, were nearly the same as the iso- $C_{p w}$ profiles, as shown in Fig. (9). On the other hand, iso-Nu profiles for all orifices in the wall jet region, $C_{p w}=0$, showed a circular shape as indicated by the line for $\mathrm{Nu}=125$ as well as that for a circular orifice. The spreads of all orifices also were nearly the same. A maximum value of $\mathrm{Nu}_{0}$ for the circular orifice was observed at $L / D_{e}=8$, which was the same value as for a typical circular nozzle ${ }^{(3)}$, but the maximum values of $\mathrm{Nu}_{0}$ for all the other orifices were obtained at $L / D_{e}=4$. Moreover, the maximum value of $\mathrm{Nu}_{0}$ increased with decreasing $n$ at $L / D_{e}=4$, and also $T_{u}$ at that position increased with decreasing like Fig. (5).

\section{CONCLUSION}

We experimentally investigated the effect of orifice configuration on flow behavior and impinging heat transfer characteristics of a jet from various regular polygonal orifices, including triangular, square, pentagonal, and hexagonal shapes.

- Axis-switching phenomenon was observed in the free jet emitted from the various polygonal orifices, and this was documented by visualizing the flow using hydrogen bubbles. The location of one complete cycle of axis switching shifted downstream of the jet with decreasing number of polygon sides.

- When the dimensionless orifice-to-target plate spacing was between 2 and 8 , the position where the contour line of the local wall pressure coefficient profile on the target plate approached a concentric circle shifted to the upstream side of the jet as the number of polygon sides increased. The shape of the local wall pressure coefficient profile after axis switching remained downstream as the number of polygon sides decreased.

- When the dimensionless orifice-to-target plate spacing was between 4 and 8 , the contour lines for the Nusselt number profile on the target plate showed the same tendency as that of the pressure coefficient profile in the impingement jet region. However, in the wall jet region, there was no remarkable difference in the contour lines for the Nusselt number profile when the number of the polygon sides was varied.

\section{NOMENCLATURE}

$C_{p w}=$ Local wall pressure coefficient on the target plate, dimensionless

$C_{p w 0}=C_{p w}$ at $y=z=0$, dimensionless

$D_{e}=$ Equivalent diameter of the orifice, $\mathrm{mm}$

$h_{a}=$ Distance from the center to the apical angle of the orifice, $\mathrm{mm}$

$h_{b}=$ Distance from the center to the base of the orifice, $\mathrm{mm}$
$L=$ Orifice-to-target plate spacing, $\mathrm{mm}$

$n=$ The number of sides on the regular polygon orifice

$\mathrm{Nu}=$ Local Nusselt number $\left(=\alpha D_{e} / \lambda\right)$, dimensionless

$\mathrm{Nu}_{0}=\mathrm{Nu}$ at $y=z=0$, dimensionless

$p=$ Static pressure on the target plate, $\mathrm{N} / \mathrm{m}^{2}$

$q=$ Heat flux on the target plate, $\mathrm{W} / \mathrm{m}^{2}$

$\operatorname{Re}=$ Reynolds number $\left(=U_{0} D_{e} / v\right)$, dimensionless

$T_{u}=$ Turbulence intensity along the $x$-direction of the free jet, $\%$

$T_{w}=$ Surface temperature on the target plate, $\mathrm{K}$

$T_{0}=$ Free-stream temperature at the orifice exit, $\mathrm{K}$

$U=$ Velocity along the $x$-direction of the free jet, $\mathrm{m} / \mathrm{s}$

$U_{0}=$ Uniform velocity at the orifice exit, $\mathrm{m} / \mathrm{s}$

$U_{m}=$ Maximum velocity along the $x$-direction of the free jet, $\mathrm{m} / \mathrm{s}$

$U_{r m s}=$ Root-mean-square of the fluctuating velocity on the $x$-direction, $\mathrm{m} / \mathrm{s}$

$x=$ Streamwise distance from the orifice exit, $\mathrm{mm}$

$y, z=$ Spatial coordinate

$y_{a}=$ Direction from the gravitational center to the apical angle of the orifice

$y_{b}=$ Direction from the gravitational center to the base of the orifice

$y_{a 1 / 2}=$ Velocity half width for the apical angle side of the orifice, $\mathrm{mm}$

$y_{b 1 / 2}=$ Velocity half width for the base side of the orifice, $\mathrm{mm}$

$\alpha=$ Local heat transfer coefficient, $\mathrm{W} / \mathrm{m}^{2} \mathrm{~K}$

$\lambda=$ Thermal conductivity of air, $\mathrm{W} / \mathrm{m} \mathrm{K}$

$v=$ Kinematics viscosity of air, $\mathrm{m}^{2} / \mathrm{s}$

$\rho=$ Density of air $\mathrm{kg} / \mathrm{m}^{3}$

\section{REFERENCES}

[1] G. N. Abramovich, The Theory of Turbulent Jets, The M.I.T Press, 1963.

[2] N. Rajaratnam, Turbulent Jets, Elsevier Scientific Pub., 1976.

[3] T. Shakouchi, Jet Flow Engineering, Morikita Publishing, 2004. (in Japanese).

[4] M. H. Steiger, and P. M. Sforza, "Further experimental results for three-dimensional free jets", AIAA Journal, Vol. 5, No. 5, pp. 800$866,1966$.

[5] F. Hussain, and H. Hussain, "Elliptic jets. Part 1. Characteristics of unexcited and excited jets", Journal of Fluid Mechanics, Vol. 208, pp. 257-320, 1989.

[6] K. B. M. Q. Zaman, "Axis switching and spreading of an asymmetric Jet: the role of coherent structure dynamics", Journal of Fluid Mechanics, Vol. 316, pp. 1-27, 1996.

[7] J. Mi, G. J. Nathan, and R. E. Luxton, "Centreline mixing characteristics of jets from nine differently shaped nozzles", Experiments in Fluids, Vol. 28, pp. 93-94, 2000.

[8] M. Hiwada, J. Mimatsu, K. Tanaka, K. Oyakawa, T. Kodera, and N. Hirose, "Effect of tone-excitation on heat transfer characteristics of an elliptic impinging jet with several aspect ratio", Proceeding 
of Thermal Engineering Conference JSME, pp. 441-442, 2001. (in Japanese).

[9] W. R. Quinn, "Near-field measurements in a equilateral triangular turbulent freejet", AIAA Journal, Vol. 43, No. 12, pp. 2574-2585, 2005.
[10] M. Chaudhari, B. Puranik, and A. Agrawal, "Effect of orifice shape in synthetic jet based impingement cooling", Experimental Thermal and Fluid Science, Vol. 34, pp. 246-256, 2010.

(C) Kanamori et al.; Licensee Bentham Open.

This is an open access article licensed under the terms of the Creative Commons Attribution Non-Commercial License (http://creativecommons.org/ licenses/by-nc/3.0/), which permits unrestricted, non-commercial use, distribution and reproduction in any medium, provided the work is properly cited. 